\title{
Synthesis and characterization of conducting poly (1-aminonaphthalene), poly (2-aminonaphthalene) and poly (aniline-co-1-aminonaphthalene)
}

\author{
BIDHAN C ROY, MAYA DUTTA GUPTA, LEENA BHOWMIK and JAYANTA K RAY* \\ Department of Chemistry, Indian Institute of Technology, Kharagpur 721 302, India
}

MS received 21 April 2003; revised 19 July 2003

\begin{abstract}
Poly (1\&2)-aminonaphthalene and poly (aniline-co-1-aminonaphthalene) have been synthesized in high yields by chemical oxidative polymerization method. The polymers are soluble in polar solvents such as DMSO, NMP etc. In PNA-2 as head-to-tail coupling cannot occur, the electrical conductivity is lower than PNA-1. The copolymer exhibits distinct morphology, higher viscosity, characteristic exciton peak, appreciable thermal stability and electrical conductivity compared to PNA-1.
\end{abstract}

Keywords. Aminonaphthalene; conductivity; thermal stability; co-polymer.

\section{Introduction}

Conjugated aromatic organic polymers e.g. polyphenylene, polyaniline, polythiophene (Sanechika et al 1979; Sorillon and Garnier 1982; MacDiarmid et al 1987a,b), have attracted a great deal of attention as electrically conducting materials. Among these organic polymers, polyaniline has attracted special attention due to its high stability towards air and moisture, high electrical conductivity (MacDiarmid et al 1985) and unique redox properties (Moon et al 1993). However, polyaniline is almost insoluble in common organic solvents especially in doped form, which causes difficulties in determination of molecular structure and restricts them to practical use. The soluble polyanilines (Angelopoulos et al 1988; Inoue et al 1989, 1991) have great advantage for characterization and preparation for the casting film (Watanabe et al 1987; Pandey et al 1993; Zheng et al 1994) which are suitable materials for practical use (Yue et al 1991). This technique has been applied in more diverse fields of research to get better applicable materials. Poly ( $N$-phenyl1-naphthaleneamine) (Wei et al 1995), poly (1-aminonaphthalene) and poly (1-aminoanthracene) (Chen and Hwang 1995) are new significant members in this field. These polynuclear aromatic amines are a new class of conducting materials, those showing good electrical conductivity without any doping due to small intrinsic band gap. Such a scenario may possibly be utilized to avoid the process of doping which is cumbersome and involves the use of corrosive materials. It is observed that in the undoped state some of these polymers have significant electrical current carrying capability, five to six orders

*Author for correspondence more than PAN base. Poly nuclear aromatic amine homopolymers have been prepared electrochemically and by oxidative methods, using $\mathrm{H}_{2} \mathrm{O}_{2}-\mathrm{Fe}$ catalyst. In the present work, we prepared poly (1-aminonaphthalene) (PNA-1), poly (2-aminonaphthalene) (PNA-2), and its co-polymer (PANA).

\section{Experimental}

Aniline (99.5\% Aldrich) was distilled twice under reduced pressure. Reagent grade ammonium persulfate $(99 \%$ E. Merck) was used as received. 1-Aminonaphthalene (Fluka) and 2-aminonaphthalene (Fluka) were used as received. 1-Aminonaphthalene and 2-aminonaphthalene were dissolved in aqueous $1 \mathrm{M} \mathrm{HCl}$ solution followed by addition of $\left(\mathrm{NH}_{4}\right)_{2} \mathrm{~S}_{2} \mathrm{O}_{8}$ solution dropwise with vigorous stirring over a period of $20-30 \mathrm{~min}$, at $30-35^{\circ} \mathrm{C}$ (Ray et al 1989; MacDiarmid et al 1991). The resultant mixture was further stirred for about $6 \mathrm{~h}$ at $30^{\circ} \mathrm{C}$. The obtained polymer was filtered, washed successively with $1 \mathrm{M}$ $\mathrm{HCl}$ and ethanol until the filtrate became colourless. Schematic representation of polyaniline and its derivatives are given in figure 1.

Co-polymer of aniline and 1-aminonaphthalene was prepared by using different mole ratios of two components in the feed. 1-Aminonaphthalene/aniline co-monomer solution was prepared by dissolving aniline and 1-aminonaphthalene in $200 \mathrm{ml} 1 \mathrm{M} \mathrm{HCl}$. $\left(\mathrm{NH}_{4}\right)_{2} \mathrm{~S}_{2} \mathrm{O}_{8}$ solution was added dropwise with constant stirring over a period of $6 \mathrm{~h}$ at $25-27^{\circ} \mathrm{C}$. The blue polymers were filtered, successively washed with $1 \mathrm{M} \mathrm{HCl}$ and ethanol and dried in vacuum over $\mathrm{P}_{2} \mathrm{O}_{5}$. The percentage yields for PNA-1, PNA-2 and $\alpha$-A were 77, 75 and 70, respectively.

The finely ground polymer powder was used for IR spectra in $\mathrm{KBr}$ pellets by Perkin Elmer spectroscope 
Table 1. Experimental details of conductivity and viscosity of polymer.

\begin{tabular}{|c|c|c|c|c|c|}
\hline Sample & Mole ratio & $\begin{array}{c}\text { Reaction } \\
\text { temp. }\end{array}$ & Yield & Conductivity & $\begin{array}{l}\eta_{\text {int }}(\mathrm{dl} / \mathrm{g}) \\
\text { in } \mathrm{H}_{2} \mathrm{SO}_{4}\end{array}$ \\
\hline Poly (1-aminonaphthalene) ( $\alpha$-PNA) & - & $30-35$ & 70 & $1.9 \times 10^{-4}$ & $0 \cdot 109$ \\
\hline Poly (2-aminonaphthalene) ( $\beta$-PNA) & - & $35-40$ & 60 & $6 \cdot 5 \times 10^{-6}$ & $0 \cdot 070$ \\
\hline Poly (aniline-co-1-aminonaphthalene) (PANA-20) & $0 \cdot 20$ & $25-27$ & 80 & $7.9 \times 10^{-7}$ & $0 \cdot 273$ \\
\hline Poly (aniline-co-1-aminonaphthalene) (PANA-35) & $0 \cdot 35$ & $25-27$ & 78 & $3.5 \times 10^{-2}$ & - \\
\hline Poly (aniline-co-1-aminonaphthalene) (PANA-50) & $0 \cdot 50$ & $25-27$ & 72 & $2.9 \times 10^{-2}$ & - \\
\hline Poly (aniline-co-1-aminonaphthalene) (PANA-70) & $0 \cdot 70$ & $25-27$ & 65 & $3 \cdot 2 \times 10^{-3}$ & - \\
\hline
\end{tabular}

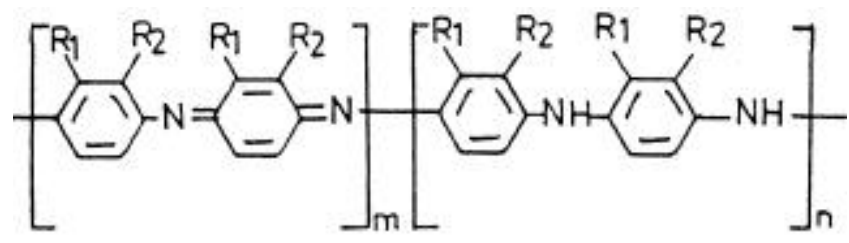

$$
\begin{aligned}
& R_{1}=R_{2}=H \text { and } \\
& R_{1}, R_{2}=-C H=C H-C H=C H-
\end{aligned}
$$

Figure 1. Structure of polymer.

(Model 883). The UV-vis spectra of polymer solution in DMSO was recorded by using Shimadzu UV-3100 spectrometer. The thermal analysis (TG, DTA) was done by a Shimadzu DT-40 unit, in air, with a heating rate of $10^{\circ} \mathrm{C} \mathrm{m^{-1 }}$. Conductivity of $\mathrm{HCl}$ doped polymers by using pellet was measured by two-probe technique employing the Philips PR 9500 unit at room temperature. Scanning electron micrographs were taken on a cam scan2 DV unit. All the measurements except conductivity were performed on base form of the polymers.

\section{Results and discussion}

Polymerization of $1 \& 2$-aminonaphthalene and co-polymerization of aniline with 1-aminonaphthalene were performed with ammonium persulfate as oxidant to get high yields. The polymers are soluble in common polar solvents such as DMSO and NMP, moderately in DMF, THF and completely soluble in $\mathrm{H}_{2} \mathrm{SO}_{4}(96 \%)$. The intrinsic viscosity (in $96 \% \mathrm{H}_{2} \mathrm{SO}_{4}$ ) qualitatively indicates that the prepared polymers have rather low molecular weight, especially poly (2-aminonaphthalene) compared to polyaniline $(0.57 \mathrm{dl} / \mathrm{g})$, which shows much higher viscosity.

The infrared spectra are shown in figures 2 and 3 . The characteristic skeletal vibration at 1586 and $1496 \mathrm{~cm}^{-1}$ of polyaniline was due to quinoid and benzoid ring stretch. Above-mentioned stretching appears at 1586 and $1503 \mathrm{~cm}^{-1}$ for PNA-1, 1618 and $1503 \mathrm{~cm}^{-1}$ for PNA-2 and 1585 and $1498 \mathrm{~cm}^{-1}$ for PANA (Chain and MacDiarmid 1986; MacDiarmid et al 1987a,b; Wei et al 1995). The IR spectra of (table 2) PANA and PNA-1 show a mixed feature with side chain oxidation peak at 1651 and $1653 \mathrm{~cm}^{-1}$ having

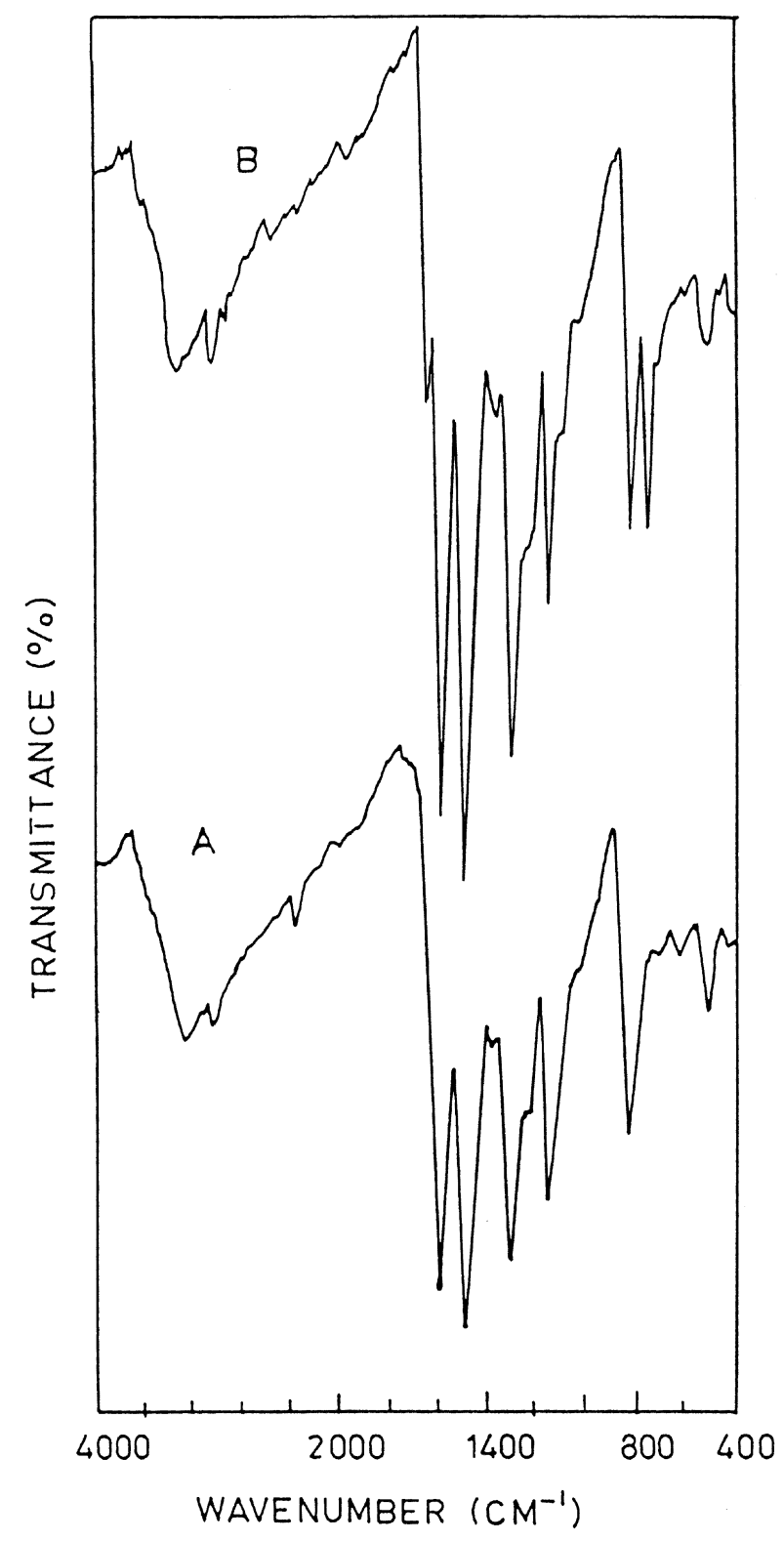

Figure 2. Infrared spectroscopy of A. PNA-1 and B. PNA-2.

relatively lesser intensity, whereas benzoid peak is relatively stronger. The distinct character of the polymer is also indicated by the almost equal intensity of the out of plane aromatic $\mathrm{C}-\mathrm{H}$ bond vibration at $814-847 \mathrm{~cm}^{-1}$ and 
Table 2. Characteristic IR stretching frequency of polymers.

\begin{tabular}{lcccccc}
\hline Polymer & $\begin{array}{c}\text { Quinoid } \\
{[\mathrm{Q}]\left(\mathrm{cm}^{-1}\right)}\end{array}$ & $\begin{array}{r}\text { Benzenoid } \\
{[\mathrm{B}]\left(\mathrm{cm}^{-1}\right)}\end{array}$ & {$[\mathrm{Q}] /[\mathrm{B}]$} & $\mathrm{C}-\mathrm{N}\left(\mathrm{cm}^{-1}\right)$ & $\mathrm{C}-\mathrm{H}\left(\mathrm{cm}^{-1}\right)$ & $\mathrm{Other}$ \\
\hline Poly (1-aminonaphthalene) & 1585 & 1498 & 0.93 & 1289 & $847 / 763$ & 1653 \\
Poly (2-aminonaphthalene) & 1618 & 1503 & $1 \cdot 11$ & 1345 & $814 / 750$ & - \\
Poly (aniline-co-1-aminonaphthalene) (PANA-20) & 1589 & 1500 & 0.96 & 1301 & $829 / 761$ & 1651 \\
\hline
\end{tabular}

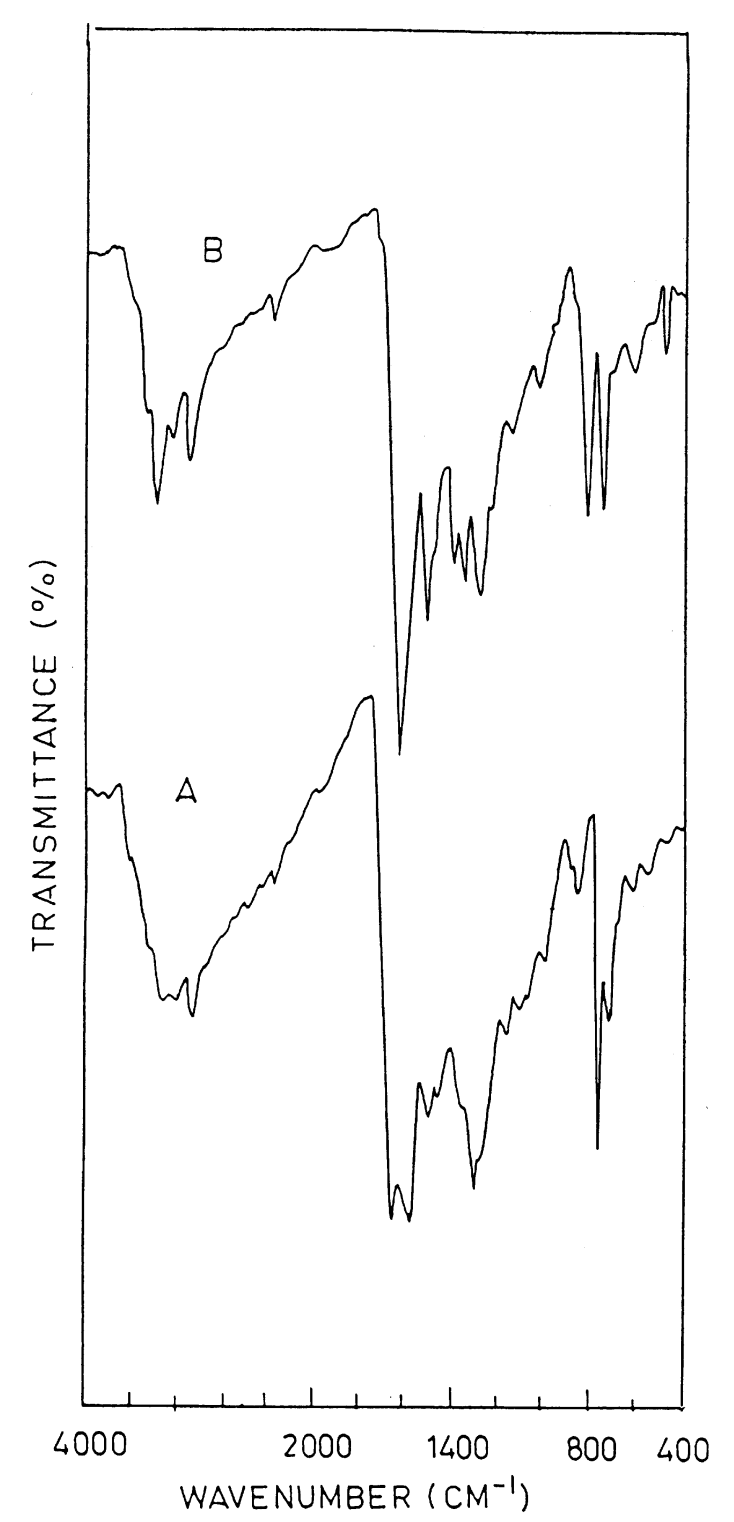

Figure 3. Infrared spectroscopy of A. PANA-20 and B. PANA-35.

$750-768 \mathrm{~cm}^{-1}$ for two and four adjacent hydrogen $(2 \mathrm{H}$ and $4 \mathrm{H}$ ) atoms. Several authors have suggested different coupling mechanisms. In the electrochemical polymerization of 1-aminonaphthalene, $\mathrm{C}-\mathrm{C}$ (tail to tail), $\mathrm{N}-\mathrm{N}$ (head to head) and $\mathrm{N}-\mathrm{C}$ (head to tail coupling) modes have been envisaged. However, it is found that in aqueous medium head to tail coupling was more dominant. In $\beta$ -

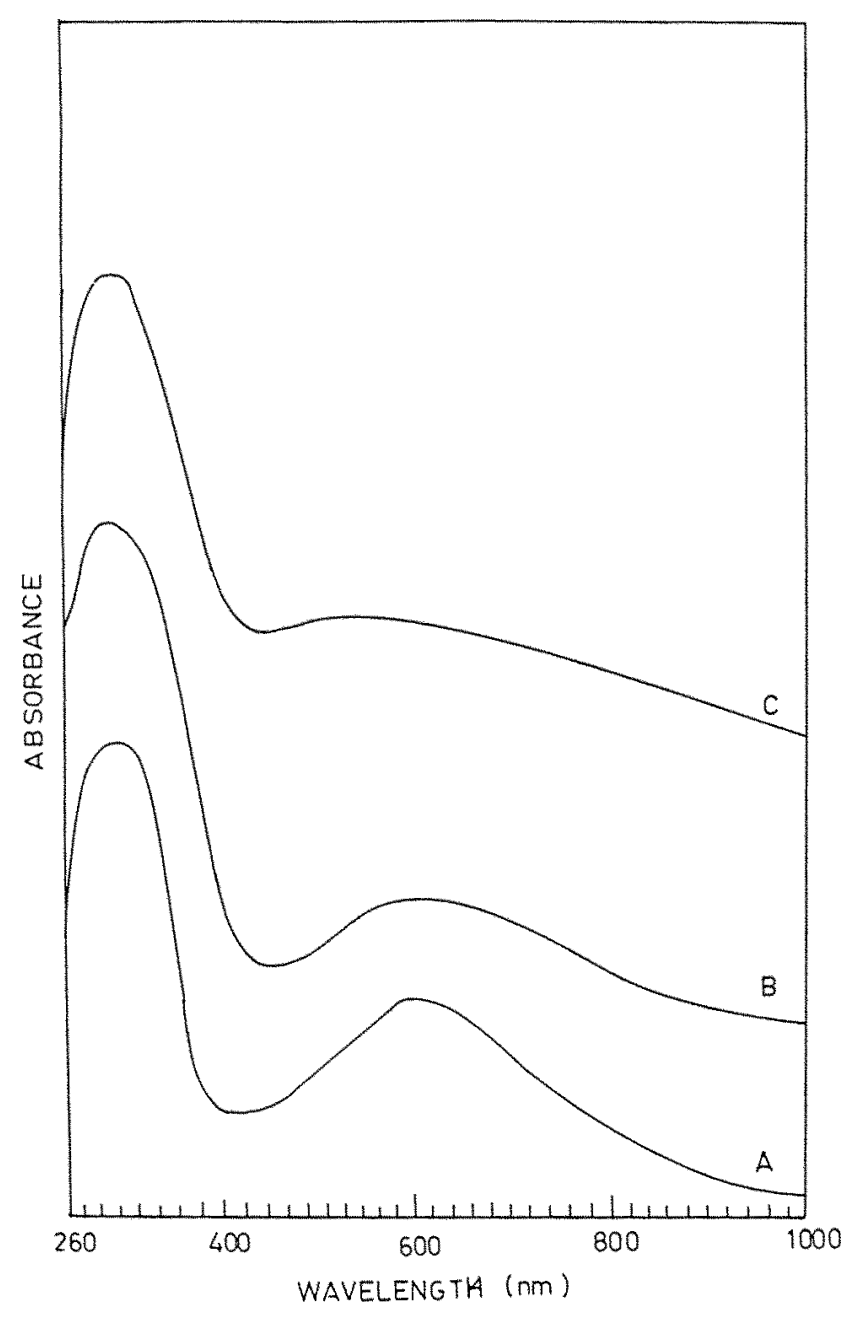

Figure 4. UV-vis absorption spectra of A. PNA-1, B. PNA-2 and C. PANA.

PNA, head to tail coupling cannot occur, the out of plane aromatic $\mathrm{C}-\mathrm{H}$ bond absorption for adjacent hydrogen (4H) (Chan et al 1993; Nguyen et al 1994) is rather reduced compared to $2 \mathrm{H}$ peak, indicating polymerization in $2: 5$ or $2: 8$ position in addition to $1: 2$ position, too much steric strain being associated with the latter.

The UV-vis absorption spectra of the polymers are shown in figure 4. PNA-1, PNA-2 and PANA display major absorption bands in DMSO at 314, 297 and $307 \mathrm{~nm}$, respectively due to $\Pi-\Pi^{*}$ transition. Bands at $495 \mathrm{~nm}$ 
Table 3. Thermal analysis of naphthalene based polymer.

\begin{tabular}{|c|c|c|c|c|}
\hline \multirow[b]{2}{*}{ Samples } & \multicolumn{3}{|c|}{ Mass loss (\%) } & \multirow{2}{*}{$\begin{array}{c}\text { Range of } \\
\text { decomposition } \\
\left({ }^{\circ} \mathrm{C}\right)\end{array}$} \\
\hline & $200^{\circ} \mathrm{C}$ & $400^{\circ} \mathrm{C}$ & $600^{\circ} \mathrm{C}$ & \\
\hline Poly (1-aminonaphthalene) base & 0 & $7-8$ & 80 & $320-630$ \\
\hline Poly (2-aminonaphthalene) base & $11-12$ & $22-23$ & 78 & $300-700$ \\
\hline $\begin{array}{l}\text { Poly (aniline-co- } 1 \text {-amino- } \\
\text { naphthalene) base }\end{array}$ & 0 & $2-3$ & 34 & $250-920$ \\
\hline
\end{tabular}
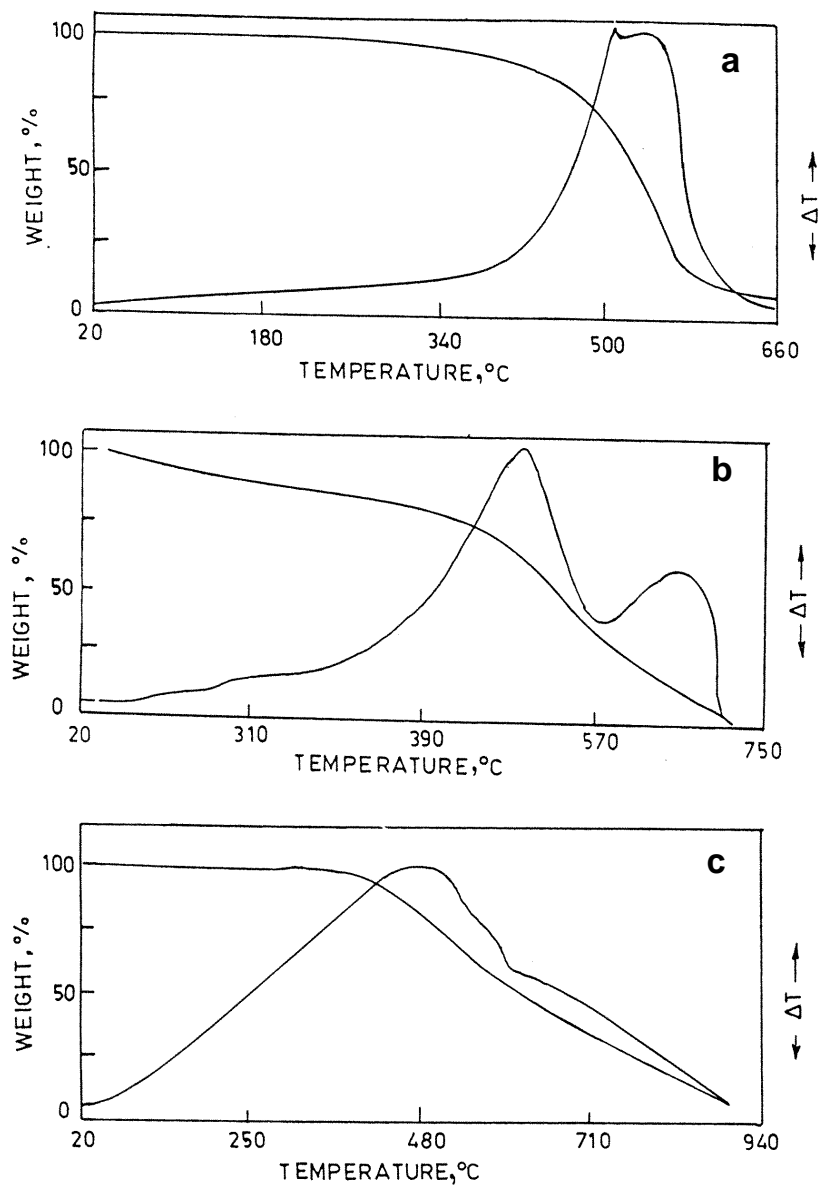

Figure 5. TG-DTA thermograms of a. PNA-1, b. PNA-2 and c. PANA.

and $575 \mathrm{~nm}$ for exciton transition appeared for PNA-1, PANA, respectively, the latter one is taken as a measure of extended conjugation in the polymer backbone. The blue shift increases with increasing proportion of 1-aminonaphthalene in the feed.

TG-DTA analysis of polymer is shown in figure 5 . Thermal data suggest that PANA is the most thermally stable polymer. $\alpha$-PNA shows about $7-8 \%$ mass loss at $400^{\circ} \mathrm{C}$ and then begin to undergo oxidative degradation, which is fast completed $\left(600^{\circ} \mathrm{C}\right)$. The exothermic change taking place in the copolymer without any accompanying mass loss up to $400^{\circ} \mathrm{C}$ is due to interchanged crosslinking reaction. An exothermic behaviour occurs above
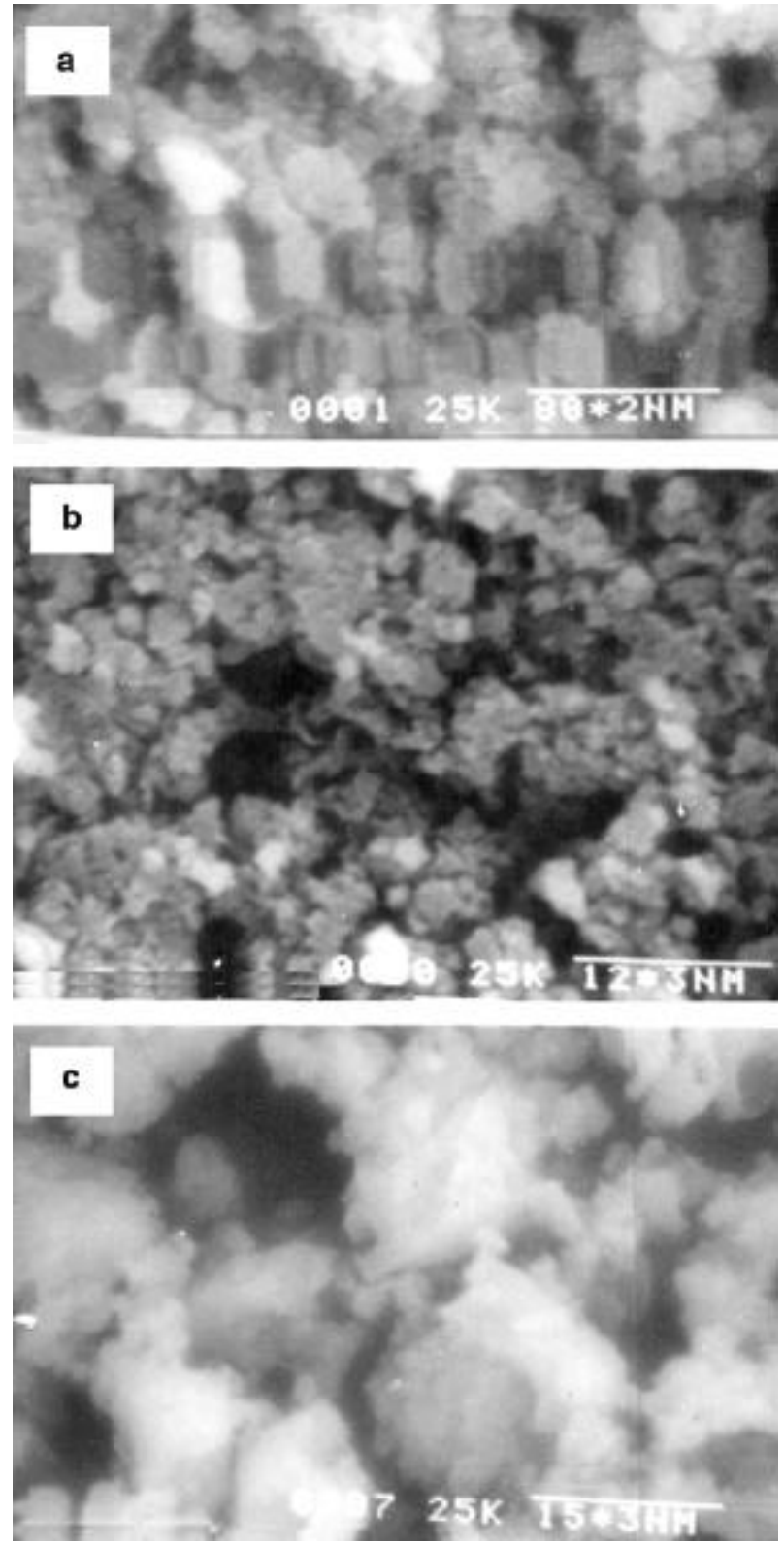

Figure 6. SEM showing a. poly (1-aminonaphthalene), b. poly (2-aminonaphthalene) and c. poly (aniline-co-1-aminonaphthalene).

$200^{\circ} \mathrm{C}$ supporting interchain cross-linking. This crosslinking can be attributed to the coupling of two neighbouring $\mathrm{N}=\mathrm{Q}=\mathrm{N}$ groups (Chen and Lee 1995). The final thermal oxidative degradation in $\beta$-PNA takes place in 
two stages at $495^{\circ} \mathrm{C}$ and $663^{\circ} \mathrm{C}$, assuming coupling occurs in $2: 5$ or $2: 8$ position apart from the $1: 2$ position. First degradation may be assigned to the polymer unit with coupling in $1: 2$ positions. Energy expulsion again indicates those with $2: 5$ and $2: 8$ coupling to be of relatively low population.

The scanning electron micrographs (figure 6) of polymers, PAN and $\alpha$-PNA, show the surface as made up of an aggregate of many small granules. The copolymer has a distinct criss-cross canal like feature, evidently absent in PAN and $\alpha$-PNA. $\beta$-PNA shows partly a more regular canal like feature, the rest being irregular, indicating more than one phase.

Conductivity measurements (table 1) show that the polymer hydrochlorides are much less conducting than polyaniline. The co-polymer exhibits appreciably higher electrical conductivity $\left(7.9 \times 10^{-2}-3.2 \times 10^{-3} \mathrm{~S} / \mathrm{cm}\right)$ compared to PNA-1 $\left(1 \cdot 1 \times 10^{-4} \mathrm{~S} / \mathrm{cm}\right)$. Low electrical conductivity of PNA-2 indicates considerable steric strain and absence of usual head to tail coupling pattern, prevailing in PNA-1.

\section{References}

Angelopoulos M et al 1988 J. Mol. Cryst. Liq. Cryst. 160151 Chan H S O, Ng S C, Sim W S, Seow S H, Tan K L and Tan B T G 1993 Macromolecules 26144

Chain J C and MacDiarmid A G 1986 Synth. Met. 13193

Chen S A and Hwang G W 1995 J. Am. Chem. Soc. 11710055

Chen S A and Lee H T 1995 Macromolecules 263254

Inoue M, Navorro R F and Inoue M B 1989 Synth. Met. 30 199
Inoue M, Brown F, Munoz I C and Ofelia F 1991 Polym. Bull. 26403

MacDiarmid A G, Mu S L, Samasiri M L D and Wu W 1985 Mol. Cryst. Liq. Cryst. 121187

MacDiarmid A G, Yang L S, Huang W S and Humphrey B P 1987a Synth. Met. 18393

MacDiarmid A G, Chiang J C, Richter A F, Samasiri M L D and Epstein A J 1987b Conducting polymers (ed.) A Alcacer (Dordrecht: Riedel Publications) pp 105-120

MacDiarmid A G, Manohar S K, Masters J G, Sun Y and Weiss H 1991 Synth. Met. 41621

Moon D K, Ezuka M, Maruyama T, Osakada K and Yamamoto T 1993 Macromolecules 26364

Nguyen M T, Kasai P, Miller J L and Diaz A F 1994 Macromolecules 273625

Pandey S S, Annapoorni S and Malhotra B D 1993 Macromolecules 263190

Ray A, Asturies G E, Kershner D L, Richter A F, MacDiarmid A G and Epstein A J 1989 Synth. Met. 29 E141

Sanechika K, Yamamoto T and Yamamoto A 1979 Polym. Prep. Jpn $\mathbf{2 8} 966$

Sorillon G and Garnier F J 1982 Electroanal. Chem. Interf. Electrochem. 135173

Sun Y, MacDiarmid A G and Epstein A J 1990 J. Chem. Soc. Chem. Commun. 529

Watanabe A, Mori K, Iwasaki Y and Nakamura Y $1987 \mathrm{~J}$. Chem. Soc. Chem. Commun. 3

Wei X L, Wang Y Z, Long S M, Bobeczko C and Epstein A J 1995 J. Am. Chem. Soc. 1182545

Yue J and Epstein A J 1990 J. Am. Chem. Soc. 1122800

Yue J, Wang Z H, Cromack K R, Epstein A J and MacDiarmid A G 1991 J. Am. Chem. Soc. 1132665

Zheng W Y, Levon K, Laakso J and Osterholm J E 1994 Macromolecules 277754 